\title{
The efficiency of chitosan-graphene oxide composite membranes modified with genipin in fuel cell application
}

\author{
Selestina Gorgieva ${ }^{1,2^{*}}$, Azra Osmić ${ }^{1}$, Boštjan Genorio ${ }^{3}$, Viktor Hacker $^{4}$, Sigrid Wolf ${ }^{4}$ and Silvo Hribernik ${ }^{1,2}$ \\ ${ }^{1}$ Faculty of Electrical Engineering and Computer Science, University of Maribor, Koroška cesta 46, 2000 Maribor, Slovenia \\ ${ }^{2}$ Faculty of Mechanical Engineering, University of Maribor, Smetanova 17, 2000 Maribor, Slovenia \\ ${ }^{3}$ Faculty of Chemistry and Chemical Technology, University of Ljubljana, Večna pot 113, SI-1000 Ljubljana, Slovenia \\ ${ }^{4} \mathrm{Graz}$ University of Technology, Institute of Chemical Engineering and Environmental Technology Inffeldgasse 25/C, 8010 Graz, \\ Austria
}

\begin{abstract}
The paper reports on the processing of chitosan N-doped reduced graphene oxide (CS/N-rGO) nanocomposite membranes prepared by a facile, dispersion-casting procedure aimed as anion exchange membranes in fuel cells. Genipin (GEN) was used as a crosslinking agent to ameliorate mechanical weakens of nanocomposite membranes, while the N-rGO filler, aside of its role as a mechanical enhancer, is expected to improve the ionic conductivity of membranes. The resulting properties of processed membranes in terms of morphology, tensile strength, elasticity, and ethanol permeability were examined. The relevance of the membranes in terms of efficiency and performance was demonstrated in a single cell test.
\end{abstract}

\section{Introduction}

As promising technology for (chemical to electrical) energy conversion, with extremely low pollutant emissions and environmental impact, the fuel cells are on the right track to dominate relevant market segments. Yet, improvements in efficiency/cost ratio of fuel cell components and the whole set-up are still necessary to reach market competitiveness against current technologies for energy production, such as the internal combustion engine and the gas turbine. Anion exchange membranes (AEMs) are among the core components of the alkaline fuel cell, acting as separators among anode and cathode and transporters of $\mathrm{OH}$ - ions. In seeking sustainability and green development, the investigation on biopolymeric AEMs nowadays becomes actual among the research community. [1] Natural polymers, such as chitosan (CS), have been recognised as a potential alternative to synthetic AEMs. [2] CS is a deacetylated chitin product found in the exoskeleton of invertebrates and the cell walls of fungi, consisting predominantly of 2-amino-2-deoxy-Dglucopyranose units, linked by $\beta-1,4$-bond. Aside from the reported attributes of this material in fuel cell applications, its mechanical weakness and swelling related to $\mathrm{OH}$ groups abundance and lack of ion-transporting moieties seek its augmentation into a more complex molecular arrangement. Our approach introduces an inorganic component, N-doped reduced graphene oxide (N-rGO), combined with genipin (GEN)-crosslinking chemistry. GEN is a natural and biodegradable alternative to synthetic crosslinking agents which reacts with primary and secondary amines [3], offering improved mechanical, chemical and thermal stability of the material. [4]

\section{Methods}

\subsection{Membrane preparation}

Neat CS membranes were prepared according to our recently published approach. [2] For the preparation of $\mathrm{CS} / \mathrm{GEN}$ and CS/GEN/N-rGO membranes, the $1 \mathrm{wt} \%$ GEN solution in ethanol and $1 \% \mathrm{~N}$-rGO dispersion were respectively added to $25 \mathrm{~mL}$ of $1 \% \mathrm{CS}$ dispersion, to final concentration of $0.002 \%$ (denominated as $100 \mu \mathrm{L}), 0.004$ $\%(200 \mu \mathrm{L}), 0.020 \%(500 \mu \mathrm{L})$ and $0.040 \% \mathrm{w} / \mathrm{v}(1000 \mu \mathrm{L})$ for GEN and $0.01 \%, 0.04 \%$ and $0.07 \% \mathrm{w} / \mathrm{v}$ for N-rGO. Final dispersion was vortexed at $3000 \mathrm{~min}^{-1}$ to obtain a homogeneous dispersion. The membranes were dried onair and stored for future characterisations.

\subsection{Attenuated Total Reflectance Fourier Transform Infrared Spectroscopy (ATR-FTIR)}

ATR-FTIR spectra were obtained on a Spectrum One FTIR spectrometer (Perkin-Elmer) with a Golden Gate ATR attachment and a diamond crystal for all components and CS membranes, containing a variable concentration of $\mathrm{N}-\mathrm{rGO}$ and GEN. The absorbance measurements were recorded within the $650-4000 \mathrm{~cm}^{-1}$ region, with 16 scans at a resolution of $4 \mathrm{~cm}^{-1}$.

\footnotetext{
* Corresponding author: selestina.gorgieva@um.si
} 


\subsection{Mechanical measurements}

The membranes' tensile strength (MPa) and elastic elongation (\%) were determined by testing machine Shimadzu, AG-X plus $10 \mathrm{kN}$. Membranes samples with specimen dimensions: $10 \mathrm{~mm} \times 20 \mathrm{~mm}$ were vertically mounted. The effective clamping distance was $25 \mathrm{~mm}$. Application of tensile force (10 kN load cell) proceeded at $1 \mathrm{~mm} \mathrm{~min}^{-1}$. Prior to testing, the membranes were equilibrated in distilled water.

\subsection{Scanning Electron Microscopy (SEM)}

The field eFE-SEM analysis was performed using a Carl Zeiss FE-SEM SUPRA 35 VP electron microscope to visualise membranes' microstructure. Imaging was performed at $1 \mathrm{kV}$ accelerating voltage at an approximately $4.5 \mathrm{~mm}$ working distance. The membranes were attached to aluminium sample holders via conductive carbon adhesive tape. Before analysis, a layer of palladium was sputtered on the surface of membrane samples.

\subsection{Ethanol permeability}

Ethanol permeability through $1 \%$ CS membrane with different $\mathrm{N}-\mathrm{rGO}$ and GEN was measured in a temperature probe at $25{ }^{\circ} \mathrm{C}$ in two temperature-controlled diffusion cells. The membrane was located between the two temperature-controlled diffusion cells (the volume of each cell unit is $25 \mathrm{~mL}$ ). Reservoir A was filled with $25 \mathrm{~mL}$ of $6 \mathrm{M} \mathrm{KOH}$; reservoir B was filled with $25 \mathrm{~mL}$ of $2 \mathrm{M}$ $\mathrm{EtOH} / 6 \mathrm{M} \mathrm{KOH}(8.5 \mathrm{w} / \mathrm{w} \%$ ethanol). To create the same pressure on the membrane, both reservoirs were filled simultaneously, as this represents the natural working environment of the fuel cell. The ethanol concentration was determined using a conductometer at different time intervals. The permeability coefficient of ethanol, $P\left(\mathrm{~cm}^{2}\right.$ $\left.s^{-1}\right)$ was calculated using the equation below.

$$
P\left(\frac{c m^{2}}{s}\right)=\frac{\left(C_{B}-C_{B 0}\right)}{\left(t-t_{0}\right)} \times \frac{V_{B} \cdot l}{A \cdot C_{A 0}}
$$

where $C_{A 0}$ represents the initial concentration of ethanol in the source compartment, and $V_{B}$ represents $\mathrm{KOH}$ volume in the receiving chamber. $A$ and $l$ represent the area and thickness of the membrane, respectively.

\subsection{Cell performance measurement (Single-cell testing, DAEFC performance)}

Before the fuel cell experiments, the membranes were doped with $\mathrm{OH}-$ by immersion in $1 \mathrm{M} \mathrm{KOH}$ for $24 \mathrm{~h}$ and extensive washing with distilled water. Electrodes were prepared as described in our previous work. [2] The Membrane Electrode Assemblies (MEAs) with $2 \times 2 \mathrm{~cm}^{2}$ were prepared by placing the membrane electrolyte between the electrodes. The MEAs were assembled in a self-constructed DAEFC. Pure oxygen gas (5.0, $99.995 \%)$ with a constant flow rate of $25 \mathrm{~mL} \min 1$ was used as a cathode feed gas. A mixture of $1 \mathrm{M}$ ethanol in $1 \mathrm{M} \mathrm{KOH}$ served as anode fuel $\left(5 \mathrm{~mL} \mathrm{~min}^{-1}\right)$. The membranes were tested additionally in $3 \mathrm{M} \mathrm{EtOH} / 5 \mathrm{M} \mathrm{KOH}$ to determine the influence of the fuel concentration on the cell performance. To evaluate the temperature dependence, experiments were conducted at room temperature, as well as at $35^{\circ} \mathrm{C}, 43{ }^{\circ} \mathrm{C}, 50{ }^{\circ} \mathrm{C}$ and $57^{\circ} \mathrm{C}$. The current densities (I) and cell potentials (V) of the single cells were determined using a Reference 600TM Potentiostat/Galvanostat/ZRA from Gamry instruments. The results were plotted in a current density-potential diagram, indicating the power density.

\section{Results and discussion}

Due to the fragility of thin CS membranes, especially after immersion in water or water $/ \mathrm{KOH}$ mixtures, the crosslinking with GEN was examined, alongside the introduction of inorganic N-rGO filler. The GEN molecule can react with the primary amino group in CS in a neutral or acidic environment, resulting in heterocyclic amines forming and developing deep-blue colour, as seen on membrane on Figure 1. The heterocyclic amines can further associate to form crosslinked networks with short (dimmer, trimer, and tetramer) bridges.



Fig. 1. Tentative scheme for GEN crosslinking in $\mathrm{NH}_{2}-$ containing polymers. Insert: CS membrane crosslinked with GEN.

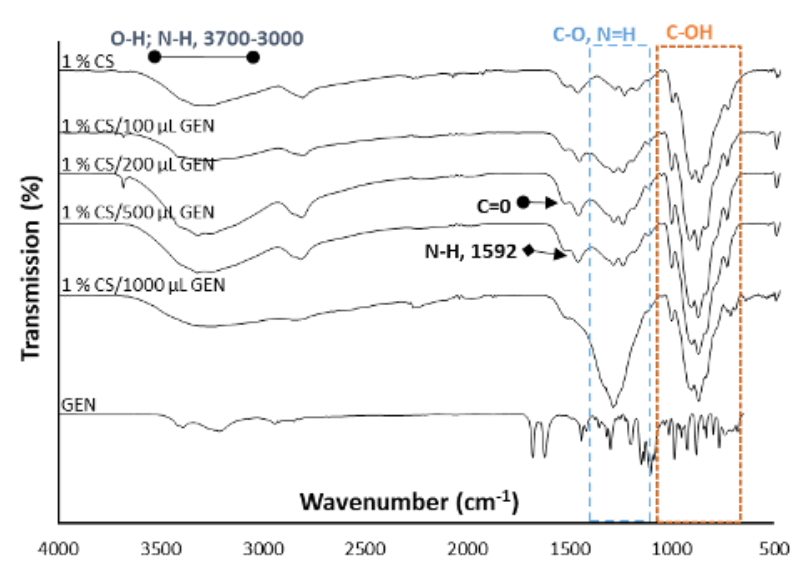

Fig. 2. ATR-FTIR spectra of CS membrane with different concentrations of GEN, including the GEN powder as control.

ATR-FTIR spectra (Figure 2) demonstrate GEN concentration-dependent spectral changes in GENcontaining membranes in comparison to neat CS membrane, which suggest on molecular interaction among the components. As explained in our previous work [5], 
two-way reaction mechanisms are possible in GEN - CS system. Herein, the spectral lines demonstrate an increase of ratio between the area of absorption bands at 1640 $1650 \mathrm{~cm}^{-1}$ and $1570-580 \mathrm{~cm}^{-1}$ assigned to $\mathrm{v}(\mathrm{C}=\mathrm{O})$ and $v(\mathrm{~N}-\mathrm{H})$, respectively, in crosslinked, relative to neat CS. Latter can be attributed to the formation of secondary amino groups among CHT amines and ester groups of GEN, being identified as a second, slower reaction under which a single, bi-functional crosslink between CHT molecules are formed. The mechanical data in Figure 3 evidence that GEN presence significantly improve the tensile strength in N-rGO - free membranes, while filler addition brings the opposite effect. In contrary, the membrane elasticity significantly decreased by GEN addition, suggesting the formation of bridges, which secures the polymer distances, restricting the membrane elongation on macro scale.

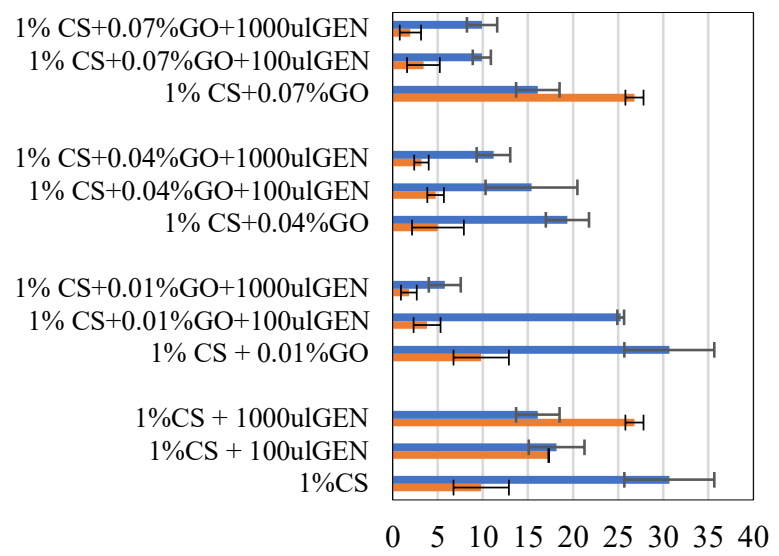

Elastic elongation (\%) $\square$ Tensile strength (MPa)

Fig. 3. Tensile strength (MPa) and elastic elongation (\%) of CS membranes with different concentrations of GEN and N-rGO.

Previous studies [6] utilising CS and rGO demonstrate that at low content, rGO can be dispersed in the polymer solution more uniformly, which may improve the adhesion with the polymer matrix, while, with the further increase of the rGO content, dispersibility within the polymer solution deteriorated, resulting in much weaker interaction with the polymer. Our results do not confirm this, and tensile strength increases with N-rGO increment in GENfree samples, suggesting that the nature of filler in terms of shape, size, and charge has a dominant influence on its efficiency as a mechanical enhancer.

SEM micrographs in Figure 4 demonstrate the smoothening effect of GEN; herein, we speculate that the presence of GEN bridges controls the membrane shrinking during the drying process, which is the leading cause for shrinking of neat CS membranes, thus positively contributing to tensile strength. Upon addition of N-rGO, the bridges may restrict the continuous assembling of filler, promoting the phase separation into condensed, $\mathrm{N}$ rGO - rich areas on one side and N-rGO - lean areas on another, forming the weak points and tensile strength reduction.
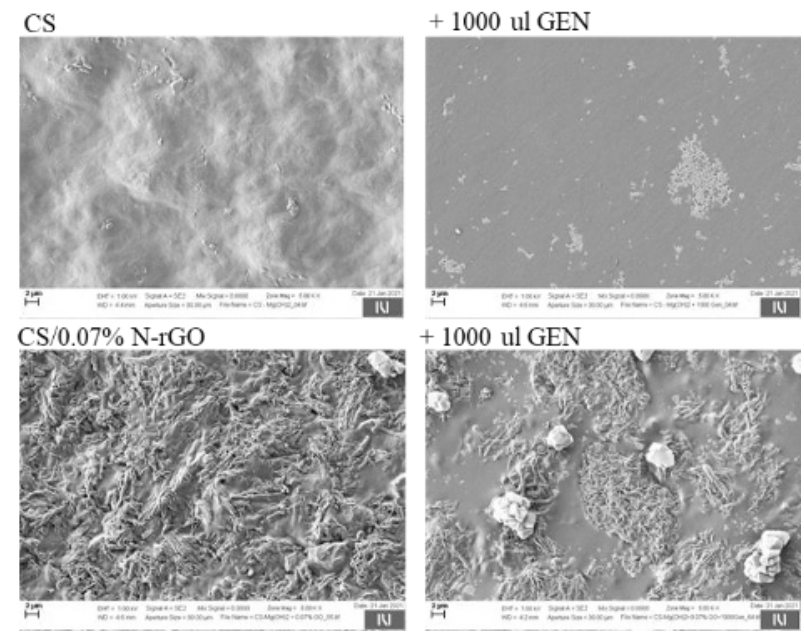

Fig. 4. ATR-FTIR spectra of CS membrane with different concentrations of GEN, including the GEN powder as control.

The fuel (ethanol) permeability is one of the critical parameters affecting the membrane performance, as the same can accelerate the degradation process of the membrane, interfere with the cathode reaction, and cause electrocatalyst poisoning. [2] It was found that the permeability was slightly increased by the addition of $\mathrm{N}$ rGO and was not affected by addition of GEN crosslinking agent, irrespective of concentration. As seen by SEM image, the fillers are expected to cause the formation of intermediate spaces in the membranes, which do not restrict the migration of ethanol molecules, resulting in increased permeability values.

The membrane performance in fuel cells is determined in direct alkaline ethanol test cells, where the effect of different loading concentrations of N-rGO and GEN concentration were investigated, as well as the influence of temperature and fuel concentration on the DAEFC membrane performance. In summary, the $\mathrm{CS} / \mathrm{N}-\mathrm{rGO}$ with the lowest graphene loading $(0.01 \%)$ at a temperature of $57{ }^{\circ} \mathrm{C}$ and the concentrated $3 \mathrm{M} \mathrm{EtOH} / 5 \mathrm{M} \mathrm{KOH}$ anode fuel presented the best overall performance (Figure 5). The best Pmax. Of $17.7 \mathrm{~mW} \mathrm{~cm}^{-2}$ could be achieved at a current density of $97.39 \mathrm{mAcm}^{-2}$.

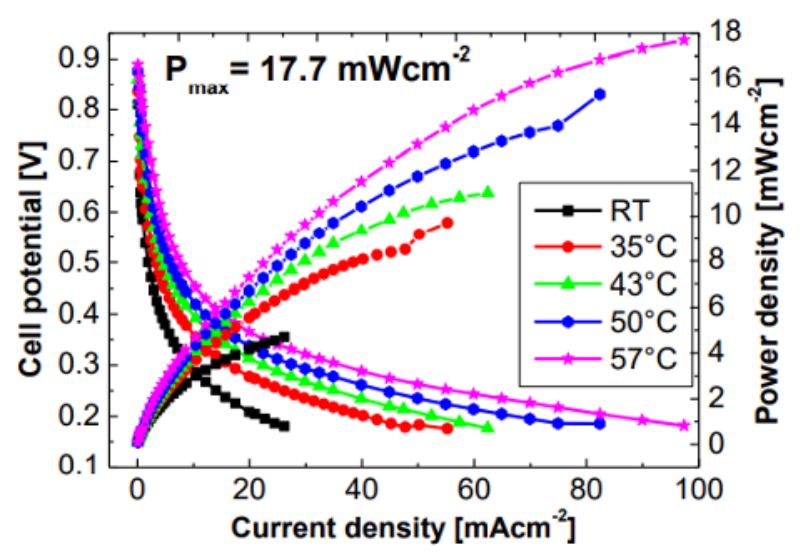

Fig. 5. DEAFC discharged cell voltage and power density of $1 \%$ $\mathrm{CS} / \mathrm{N}-\mathrm{rGO}(0.01 \%)$ membranes at different temperatures with 3 $\mathrm{M} \mathrm{EtOH} / 5 \mathrm{M} \mathrm{KOH}$. 
Very comparable are values with the addition of $100 \mathrm{ul}$ GEN, without N-rGO loading (Pmax. of $17.2 \mathrm{~mW} \mathrm{~cm}^{-2}$ at a current density of $\left.96.90 \mathrm{~mA} \mathrm{~cm}^{-2}\right)$. Latter, suggest on the potential action of GEN or GEN-product in ion conduction, as also suggested by ionic conductivity study (not presented here). Yet, the GEN presence at higher concentrations increases membrane fragility and their disintegration under a highly alkaline medium.

\section{Conclusion}

We demonstrated that CS-based nanocomposite membranes containing N-rGO and/or GEN significantly outperform the fuel cell performance of the pristine CS membrane. In previous studies [19], the highest reported power density value for CS-based membranes was 62.2 $\mathrm{mW} \mathrm{cm}{ }^{-2}$ (current density $174 \mathrm{~mA} \mathrm{~cm}^{-2}$ ) at $60^{\circ} \mathrm{C}$ and 72.7 $\mathrm{mW} \mathrm{cm}{ }^{-2}$ (current density $209 \mathrm{~mA} \mathrm{~cm}^{-2}$ ) at $80{ }^{\circ} \mathrm{C}$. Compared to these investigations, lower temperatures (57 ${ }^{\circ} \mathrm{C}$ instead of $60{ }^{\circ} \mathrm{C}$ and $80{ }^{\circ} \mathrm{C}$ ), a lower oxygen fuel rate


loading for the anode $\left(0.75 \mathrm{mg} \mathrm{cm}^{-2}\right.$ instead of $\left.2 \mathrm{mg} \mathrm{cm}^{-2}\right)$ and the cathode $\left(0.5 \mathrm{mg} \mathrm{cm} \mathrm{cm}^{-2}\right.$ instead of $\left.1 \mathrm{mg} \mathrm{cm}^{-2}\right)$ were used showing the relevance of the tested sustainable biobased membranes for use in energy conversion applications.

The authors would like to acknowledge the financial support from Slovenian Research Agency, in the frame of project "Graphene Oxide-based MEAS for the Direct Ethanol Fuel Cell « (grant number N2-0087), the Textile Chemistry Program (P20118) and from the Austrian Science Fund (FWF) under project number I 3871-N37.

\section{References}

1. M. Hren, M. Božič, D. Fakin, K.S. Kleinschek, S. Gorgieva, Alkaline membrane fuel cells: Anion exchange membranes and fuels, Sustain. Energy Fuels. 5 (2021) 604-637.

2. S. Gorgieva, A. Osmić, S. Hribernik, M. Božič, J. Svete, V. Hacker, S. Wolf, B. Genorio, Efficient Chitosan/Nitrogen-Doped Reduced Graphene Oxide Composite Membranes for Direct Alkaline Ethanol Fuel Cells, Int. J. Mol. Sci. 22 (2021).

3. V.G. Tacias-Pascacio, E. García-Parra, G. VelaGutiérrez, J.J. Virgen-Ortiz, Á. Berenguer-Murcia, A.R. Alcántara, R. Fernandez-Lafuente, Genipin as an emergent tool in the design of biocatalysts: Mechanism of reaction and applications, Catalysts. 9 (2019).

4. a Bigi, G. Cojazzi, S. Panzavolta, N. Roveri, K. Rubini, Stabilisation of gelatin films by crosslinking with genipin., Biomaterials. 23 (2002) 4827-32.

5. S. Gorgieva, T. Vuherer, V. Kokol, Autofluorescenceaided assessment of integration and $\mu$-structuring in chitosan/gelatin bilayer membranes with rapidly mineralised interface in relevance to guided tissue regeneration, Mater. Sci. Eng. C. 93 (2018) 226-241.

6. X. Qian, N. Li, Q. Wang, S. Ji, Chitosan/graphene oxide mixed matrix membrane with enhanced water permeability for high-salinity water desalination by pervaporation, Desalination. 438 (2018) 83-96. 